\title{
A Hipótese da Acessibilidade Conceitual para a Tipicidade e a Produção Lingüística
}

\section{The Hypothesis of Conceptual Accessibility for Typicality and Linguistic Production}

\author{
Gerson A. Janczura $a^{a^{*}}$, Douglas L. Nelson ${ }^{b}$

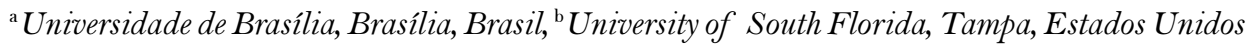

\begin{abstract}
Resumo
Este artigo estende a hipótese da acessibilidade conceitual proposta por Janczura e Nelson (1999) para a tarefa de produção lingüística e propõe um procedimento alternativo para avaliar a participação dos atributos dos conceitos em julgamentos de tipicidade. Os resultados evidenciaram que diferentes graus de tipicidade não estão relacionados à probabilidade de um atributo ser considerado como presente em um membro de uma categoria, mas podem ser previstos pela força associativa do membro em relação à categoria que pertence. Demonstrou-se, também, que a produção lingüística pode ser determinada em termos da acessibilidade dos membros de categorias na memória e que este resultado é paralelo à tarefa de jul gamentos de tipicidade. Ou seja, membros de categorias mais acessíveis na memória são considerados como melhor representantes do que menos acessíveis. Sugere-se que julgamentos de tipicidade são apoiados na memória para a informação categórica. Quanto maior for o acesso ao conhecimento que o indivíduo tiver sobre o membro ou suas características, maior será a percepção de sua representatividade. Propõe-se que, para categorias conhecidas, a tipicidade deveria ser reinterpretada como uma medida de acessibilidade ao invés de representatividade conceitual.

Palavras-chave: Acessibilidade conceitual; tipicidade; força associativa; produção lingüística; conceitos.

Abstract

This paper extends the accessibility hypotheses proposed by Janczura and Nelson (1999) to linguistic production and presents a procedure for evaluating the participation of attributes in typicality judgments. The results show that different degrees of typicality are not related to the probability of an attribute being considered a part of an exemplar but can be predicted by the strength of association between an instance and its category. It also demonstrates that accuracy in a linguistic production task can be explained in terms of the accessibility of category members in memory. These results parallel typicality judgments. That is, more accessible members in memory are considered better category exemplars. It suggests that memory for category information may underlie typicality judgments. The more a subject knows a category exemplar or its features, the better it is perceived as representing the category. This paper proposes that, for known categories, typicality should be re-interpreted as a measure of category accessibility rather than category representativeness.

Keywords: Concept accessibility; tipicality; associative strenght; linguistic production; concepts.
\end{abstract}

A tipicidade dos membros de categorias influencia várias tarefas e variáveis psicológicas como a evocação semântica (Zhang \& Chen, 2002), julgamentos diagnósticos (Evans, Herbert, Nelson, Rosemary \& Gaudiano, 2002), preferências estéticas (Hekkert, Snelders \& van-Wieringen, 2003; Veryzer \& Hutchinson, 1998; Whitfield \& Slatter, 1979), verificação categórica (Kiran \& Thompson, 2003), nomeação de categorias na afasia (Kiran \& Thompson, 2003) e tempo de reação em tarefas de categorização rápida (Verbeemen, Storms \& Verguts, 2003). A tipicidade também prediz julgamentos absolutos de pertencimento categórico (Estes, 2003), e está correlacionada com a estimação média de realismo artístico (Shortess, Clarke, Craig, Richter

\footnotetext{
* Endereço para correspondência: Instituto de Psicologia/PPB, Universidade de Brasília, Asa Norte, Campus Universitário, Brasília, DF, 70910-900. E-mail: janczura@unb.br

Esta pesquisa foi apoiada pelo Conselho Nacional de Desenvolvimento Científico e Tecnológico (CNPq) - Brasil. Os autores agradecem às sugestões dos revisores cujas observações aprimoraram o presente artigo
}

\& Seay, 2000) e com o nível de nomeação de estímulos visuais (Op-de-Beeck \& Wagemans, 2001).

Usualmente, exemplos de categorias considerados como mais típicos são julgados mais rapidamente como membros de uma categoria (Rips, Shoben \& Smith, 1973), e são frequentemente produzidos e verificados com maior acurácia como pertencendo a uma categoria (Battig \& Montague, 1969). A tipicidade também influencia outras tarefas como ordem de aprendizagem, esboçar desenhos, julgamentos indutivos, produção lingüística e raciocínio dedutivo.

Tradicionalmente, o constructo tem sido tratado como um indicador da estrutura de categorias conceituais. Em geral, a tipicidade é conceitualizada como refletindo a similaridade entre os membros de uma categoria ou em relação a uma representação da categoria (Barsalou, 1985; McCloskey \& Glucksberg, 1978; Medin \& Schaffer, 1978; Rosch \& Mervis, 1975; Smith, Shoben \& Rips, 1974).

Essa explicação estrutural para a tipicidade foi desafiada, recentemente, pela hipótese da acessibilidade conceitual. 
O desafio emergiu de estudos mostrando que categorias de rima produzem julgamentos de tipicidade semelhantes às categorias semânticas (Janczura \& Nelson, 1999). Estas categorias não oferecem nenhuma base confiável para julgamentos de tipicidade em termos da similaridade estrutural porque, por definição, cada exemplo de rima é igualmente similar aos demais membros de sua categoria (Wood, 1971). Ilustrando, canto, santo, pranto, e manto são membros igualmente semelhantes e igualmente válidos da categoria de rima anto.

Entretanto, os resultados de vários experimentos revelaram que os julgamentos de tipicidade deste tipo de categoria eram paralelos aos julgamentos de categorias semânticas e foram previstos pela sua acessibilidade na memória medida através de normas de associação livre. Exemplos de categorias de rima produzidos com maior frequêencia, em resposta ao nome da categoria como pista em normas associativas, foram julgados como mais típicos do que exemplos menos freqüentes.

De acordo com a hipótese da acessibilidade conceitual, os julgamentos de tipicidade refletem a acessibilidade na memória para categorias conhecidas. Os membros de categorias que são experienciados mais frequentemente na interação entre o indivíduo e seu meio tornam-se mais acessíveis em relação às suas categorias e tendem a serem percebidos como mais típicos. Normas de associação livre estimam a força da conexão entre a categoria e seus membros (Nelson, McEvoy, \& Dennis, 2000).

Os membros que estão mais fortemente associados às categorias são considerados mais acessíveis. A acessibilidade também pode ser determinada episodicamente variando-se a frequiência de exposição do exemplo durante uma sessão experimental. Utilizando este procedimento, Janczura e Nelson (1999) verificaram que exemplos experienciados mais frequentemente durante um episódio eram percebidos como mais típicos. A hipótese da acessibilidade proporciona uma explicação direta para este resultado e é difícil de compreender como uma abordagem estrutural explicaria este dado.

Porque a frequiência da apresentação de um membro de categoria aumentaria a similaridade estrutural de categorias semânticas e de rima? As manipulações da freqüência experimental e pré-experimental da experiência sugerem que, para categorias conhecidas, a causa subjacente dos julgamentos de tipicidade deveriam ser reinterpretados. Isto é, a tipicidade poderia ser um indicativo da acessibilidade conceitual e não da representatividade conceitual. A hipótese da acessibilidade conceitual poderia prever o desempenho em outras tarefas como, por exemplo, a produção lingüística?

$\mathrm{Na}$ tarefa de produção lingüística, exemplos de categoria variam na probabilidade de serem gerados em uma sentença de acordo com a sua tipicidade. Probabilidades mais altas estão associadas com maiores índices de tipicidade (Mervis, Catlin \& Rosch, 1976; Rosch, 1975). De acordo com a hipótese estruturalista, a estrutura do estimulo é o que determina a probabilidade deste ser produzido na sen- tença. Ou seja, os resultados são explicados em termos do grau de semelhança ou superposição de características de um exemplo em relação à representação da categoria ou outros membros da mesma.

Contudo, existe a possibilidade de que o grau de semelhança seja influenciado pela lembrança dos atributos do conceito. Os atributos dos membros da categoria com maiores índices de semelhança ou maior superposição de atributos com os demais membros poderiam ser lembrados com maior probabilidade do que os atributos dos membros com semelhança inferior. Esta possibilidade já foi comentada por Murphy e Medin (1985). Hampton (1979) mostrou que o julgamento médio da importância de um atributo correlacionava-se significativamente com a frequiência de sua produção, e Ashcraft (1978) relatou que o número de propriedades geradas para membros típicos de categorias era significativamente superior do que para membros atípicos. Estes resultados sugerem que a comparação entre os graus de semelhança dos membros de categorias pode ser influenciada pela memória dos atributos da categoria.

O presente estudo argumenta que o desempenho naquelas tarefas pode revelar diferentes graus de acessibilidade dos membros das categorias na memória. Uma indicação desta possibilidade apóia-se em estudos de evocação com pista (Janczura, 1994) e pesquisas mostrando que exemplos de categorias aprendidas são produzidos diferentemente em normas de associação livre (Battig \& Montague, 1969; Nelson \& McEvoy, 1976; McEvoy \& Nelson, 1982).

O estudo de Janczura (1994) usou o paradigma da evocação com pista para examinar a relação entre a acessibilidade e julgamentos de tipicidade avaliando a probabilidade e ordem da evocação. Os resultados evidenciaram que exemplos mais fortemente associados às suas categorias eram julgados como mais típicos e eram lembrados com maior probabilidade do que exemplos mediana ou fracamente associados. Eles eram também lembrados antes do que exemplos mais fracamente associados às categorias, e exemplos lembrados antes eram associados com índices superiores de evocação.

Os estudos acima sugerem a possibilidade de que a acessibilidade conceitual possa dirigir a evocação com pista e outras tarefas como a produção lingüística. A acessibilidade poderia determinar o desempenho nestas tarefas, quando as mesmas se apóiam no acesso a exemplos de categorias fortemente aprendidas ao invés da tipicidade. De acordo com a hipótese da acessibilidade, exemplos mais acessíveis deveriam ser produzidos com maior probabilidade e serem percebidos como melhores exemplos da categoria. Neste sentido, esta hipótese é uma extensão da heurística da disponibilidade de Kahneman e Tversky (1973) e também está relacionada com os estudos que mostram os efeitos da fluência do desempenho no processamento (Begg \& Armour, 1991; Whittlesea, 1993).

A demonstração de que a acessibilidade conceitual influencia a produção lingüística não elimina, necessariamente, a participação potencial dos atributos nos julgamentos de tipicidade para categorias conhecidas. O problema é como 
medir a acessibilidade conceitual e a estrutura do estímulo de maneira compatível. Conforme foi mencionado anteriormente, a acessibilidade conceitual é indexada diretamente através de normas de associação livre. Em contraste, a maioria das medidas da estrutura conceitual está baseada nos atributos das categorias. Isto levanta questões importantes: Como os atributos deveriam ser coletados e o que deveria delimitar a definição de atributo ou característica (Medin, 1989; Murphy \& Medin, 1985)? Quais atributos ou características deveriam ser incluídos na determinação da tipicidade considerando que o contexto influencia a tipicidade dos exemplos (Barsalou, 1987; Barsalou \& Sewell, 1984; Roth \& Shoben, 1983)?

O objetivo específico dos experimentos relatados abaixo foi apresentar um procedimento alternativo para avaliar a relação entre a estrutura de categorias e acessibilidade conceitual, e avaliar a previsão da hipótese da acessibilidade conceitual para as tarefas de produção lingüística e julgamentos de tipicidade. O Experimento 1 investigou se a estrutura dos membros de categorias varia sistematicamente com diferentes níveis de força associativa. Se esta estrutura não varia em função da acessibilidade, mas a tipicidade varia, então a hipótese da acessibilidade poderá ser aceita como uma explicação razoável para os respectivos julgamentos.

No Experimento 2, os participantes foram solicitados a produzir o primeiro membro de uma categoria em uma sentença, após terem sido expostos a uma lista de membros da categoria, e a avaliar a sua tipicidade. Nas duas tarefas contrastaram-se membros que variaram no grau de força associativa em relação às categorias. A hipótese da acessibilidade conceitual sugere que membros mais fortemente associados às categorias deverão ser produzidos com maior probabilidade e julgados como mais típicos.

\section{Experimento 1}

Explicações estruturais para julgamentos de tipicidade de membros de categorias assumem que estes estão baseados nas suas respectivas estruturas. Isto é geralmente demonstrado comparando-se os atributos ou características geradas para os membros das categorias. Observa-se, usualmente, que atributos diferentes são produzidos com diferentes probabilidades e estas são interpretadas como indicando a importância de cada atributo ou característica.

Considerando que estes resultados estão apoiados na lembrança de atributos da memória é provável que, para membros de categorias mais acessíveis na memória, os indivíduos lembrem mais atributos e, em conseqüência, estes membros evidenciem maior semelhança intracategórica. Um procedimento alternativo, que consiste em apresentar o conjunto de atributos juntamente com o membro da categoria, reduz a confiança depositada sobre a dependência da memória na avaliação da importância desta informação.

Neste estudo, os participantes foram solicitados a julgar a probabilidade de uma lista de atributos serem parte de membros de categorias semânticas. Este procedimento permite a comparação dos atributos na ausência de um compo- nente forte de memória e, ainda, a avaliação das previsões da hipótese da acessibilidade para julgamentos de tipicidade.

De acordo com esta hipótese, esperava-se que não ocorreriam diferenças significativas entre os pesos estimados aos atributos em função da acessibilidade dos membros das categorias. Além disto, os julgamentos de tipicidade deveriam variar sistematicamente em função da acessibilidade conceitual, independentemente da probabilidade estimada da ocorrência dos atributos. Membros de categorias mais fortemente associados às suas categorias deverão ser julgados como mais típicos do que membros mais fracamente associados.

\section{Método}

\section{Participantes e delineamento}

Cinqüenta e oito estudantes universitários participaram voluntariamente deste experimento. $\mathrm{Na}$ tarefa de julgamento dos atributos $(\mathrm{n}=33)$ e julgamentos de tipicidade $(\mathrm{n}=$ 25) a variável força associativa foi manipulada entre-sujeitos em três níveis: forte, médio e fraco.

\section{Materiais}

A fim de selecionar materiais para este experimento, 200 universitários participaram voluntariamente de uma tarefa de listagem de atributos. A tarefa utilizou 20 categorias semânticas retiradas das normas de associação livre de Janczura (1996). Para cada categoria foram selecionados 3 exemplos cuja força associativa variava de forte até fraco sendo as respectivas médias $0,21(\mathrm{DP}=0,03)$ para membros fortemente associados, $0,11(\mathrm{DP}=0,01)$ para membros medianamente associados e $0,02(\mathrm{DP}=0,01)$ para membros fracamente associados às categorias.

Cada participante recebeu um bloco que continha 6 categorias diferentes selecionadas randomicamente do grupo de vinte categorias, e 6 exemplos de cada categoria sendo 2 de cada nível de força associativa. A primeira página do bloco apresentava as instruções e, as seis folhas seguintes continham, em cada uma, um exemplo da categoria. Os nomes da categoria seguida do exemplo estavam dispostos no cabeçalho de cada página sendo que a ordem das páginas era aleatória.

A tarefa dos participantes foi listar todos os atributos da categoria que pudessem pensar durante 90 segundos (Rosch, 1975). O tempo foi controlado pelo experimentador através de comandos verbais. A tarefa foi administrada em grupos de 22 a 37 participantes e cada categoria foi respondida por 20 indivíduos. Três juízes independentes avaliaram os atributos gerados. Os atributos considerados como não pertencentes à categoria foram excluídos. Foram, também, eliminados atributos idiossincráticos, ou seja, aqueles produzidos por apenas um participante.

Nove categorias semânticas e 3 membros de cada foram selecionados da tarefa acima para a tarefa de julgamentos de probabilidade de ocorrência dos atributos (descrito nos Procedimentos). Os membros de cada categoria eram dos 
níveis de força associativa forte, médio e fraco, respectivamente. Um total de 27 exemplos de categorias foi utilizado. As médias da força associativa para os níveis forte, médio e fraco foram 0,22 $(\mathrm{DP}=0,02), 0,10(\mathrm{DP}=0,01)$ e 0,01 $(\mathrm{DP}$ $=0,005)$, respectivamente.

Seis atributos de cada categoria foram também selecionados totalizando 54 itens. Um terço dos atributos tinha probabilidade alta de ocorrência (ou seja, os atributos eram produzidos para todos os membros da categoria), 1/3 tinha a probabilidade média (ou seja, eram produzidos para apenas 2 membros da categoria) e $1 / 3$ tinha a probabilidade baixa (ou seja, eram produzidos para somente um membro da categoria).

Os nomes das categorias, os seus membros e os atributos foram organizados em 9 blocos que incluíam o nome de uma categoria, 3 membros (um de cada nível de força associativa), e 6 atributos ( 2 de cada nível de probabilidade). Os materiais utilizados nos julgamentos de probabilidade de ocorrência podem ser vistos no Anexo A.

\section{Procedimentos}

$\mathrm{Na}$ tarefa de julgamentos de probabilidade de ocorrência dos atributos foram elaborados 3 conjuntos de estímulos contendo, cada um, 3 categorias, 3 exemplos e 6 atributos, perfazendo um total de 18 atributos por conjunto para serem avaliados. A ordem de apresentação dos atributos por categoria foi previamente sorteada e os conjuntos foram apresentados impressos em papel para os julgamentos. As categorias e seus respectivos membros e atributos foram organizados na forma de tabelas com 4 colunas. O cabeçalho continha o nome da categoria. A primeira coluna à esquerda continha os atributos e, as 3 colunas à sua direita continham espaços sublinhados para as respostas dos participantes. O topo de cada uma destas colunas incluía o membro em relação ao qual o atributo deveria ser julgado. A tarefa dos participantes era julgar a probabilidade, entre $0 \%$ e $100 \%$, de cada membro da categoria possuir cada um dos atributos relacionados. Para responder, os participantes deveriam escrever nos espaços apropriados, os números que expressassem seus julgamentos.

Considerando que a informação a ser avaliada é apresentada aos participantes, é razoável esperar que a memória para a evocação desta informação não interferirá nos julgamentos de probabilidade de ocorrência. A Figura 1, abaixo, ilustra como categorias, exemplos e atributos foram apresentados.

\begin{tabular}{llll}
\hline \multicolumn{4}{c}{ Jóia } \\
\hline & Colar & Diamante & Aliança \\
\hline cobiçado(a) & - & - & - \\
fino(a) & - & - & - \\
prata & - & - & - \\
papel & --- & ---- & ---- \\
raro(a) & ---- & ---- & ---- \\
pérola & ---- & ---- & ---- \\
\hline
\end{tabular}

Figura 1: Julgamentos de probabilidade dos atributos.
$\mathrm{Na}$ tarefa de julgamentos de tipicidade, os participantes foram solicitados a julgar a representatividade para os 27 exemplos das categorias utilizando uma escala de 7 pontos. As instruções solicitavam atribuir o valor 1 para os membros julgados como ótimos exemplos, 4 para membros medianamente representativos, e 7 para o exemplo mais pobre da categoria. Entretanto, foi destacado que qualquer número da escala poderia ser utilizado. O tempo para realizar a tarefa era livre. As categorias e seus membros foram apresentados em uma folha de papel em ordem aleatória. Os participantes deveriam responder escrevendo um número, de 1 a 7 , que melhor correspondesse aos seus julgamentos.

\section{Resultados}

Duas análises da variância foram realizadas: a primeira verificou a existência de diferenças significativas nos pesos atribuídos aos atributos em função dos 3 níveis de acessibilidade conceitual. A segundo análise verificou o efeito da força associativa nos julgamentos de tipicidade. Os pesos médios dos atributos dos membros foram agrupados segundo o nível de força associativa (forte, médio fraco) para as nove categorias. A análise fatorial univariada para medidas repetidas foi aplicada ao fator força associativa adotando-se um nível de significância de 0,05. Nenhuma diferença significativa entre as médias foi observada pela ANOVA, $\mathrm{F}(2,8)=2,796, \mathrm{p}=0,09$.

A ANOVA aplicada à tarefa de julgamento da tipicidade revelou um efeito significativo da força associativa sobre a tipicidade, $\mathrm{F}(2,8)=4,199, \mathrm{p}=0,03$. Ou seja, exemplos mais fortemente associados às suas categorias foram julgados como mais típicos $(1,90)$ do que exemplos medianamente $(2,53)$ ou fracamente associados $(3,36)$. O teste de Tukey (p $<0,05)$ revelou que as diferenças entre as médias forte e fraco foram significativas.

Os resultados deste experimento mostram que, quando se julga a probabilidade de um atributo ser considerado parte de um membro, nenhuma diferença entre os atributos é verificada em função da força que associa um membro à sua categoria. Apesar disto, os membros das categorias são percebidos como apresentando diferentes graus de tipicidade e este gradiente é uma função da força associativa deste em relação à sua categoria. Estes resultados são inconsistentes com explicações estruturais que sustentam que os atributos dos membros típicos são mais característicos no sentido de estarem presentes naqueles exemplos do que os atributos dos membros menos típicos.

Por fim, evidenciou-se que os julgamentos de tipicidade variam em função da acessibilidade dos conceitos na memória. Ou seja, membros de categorias mais acessíveis na memória tendem a serem julgados como exemplos melhores do que membros menos acessíveis. Este resultado é consistente com a hipótese da acessibilidade conceitual para julgamentos de tipicidade. 


\section{Experimento 2}

O objetivo deste experimento foi demonstrar que a acessibilidade conceitual poderia explicar o desempenho na tarefa de produção lingüística, e prever julgamentos de tipicidade. De acordo com Janczura e Nelson (1999), membros mais fortemente associados às suas categorias são mais acessíveis na memória. Assim, se o indivíduo for solicitado a produzir exemplos de categorias em sentenças será mais provável a produção de itens mais acessíveis do que menos acessíveis na memória. Além disto, membros de categorias produzidos com maior probabilidade deverão ser julgados como mais típicos.

\section{Método \\ Participantes e delineamento. \\ Trinta estudantes universitários participaram volunta- riamente do experimento. Aplicou-se um delineamento fatorial para medidas repetidas no qual manipulou-se a vari- ável força associativa em três níveis: forte, média e fraca.}

\section{Materiais}

Quinze categorias semânticas foram amostradas das normas de associação livre de Janczura (1996). Três exemplos de cada categoria, cuja força associativa variava de forte até fraca, foram selecionados perfazendo um total de 45 exemplos. A força associativa refere-se à probabilidade de um item ser produzido como membro de uma categoria em uma tarefa de associação livre. As médias dos níveis de força associativa forte, média e fraca foram, respectivamente, 0,22 (DP = 0,02), 0,10 (DP = 0,01) e 0,01 $(\mathrm{DP}=0,005)$. Utilizaram-se as seguintes categorias e membros: ferramenta de carpinteiro (serrote, pá, esmeril), flor, (margarida, orquídea, tulipa), bebida alcoólica (vinho, vodka, licor), calçado (sapato, sandália, botina), instrumento de percussão (tambor, atabaque, cuíca), mobília (cama, mesa, cômoda), cobra venenosa (coral, jararaca, quatiara), ser místico (deus, duende, guru) e raça de cachorro (pastor alemão, poodle, collie).

\section{Procedimentos}

A tarefa de produção lingüística incluía duas etapas. $\mathrm{Na}$ primeira etapa, pares de palavras contendo o nome de uma categoria e um exemplo foram apresentados randomicamente, pelo computador, durante 3 segundos cada par. A tarefa dos participantes era ler os pares em voz alta e tentar memorizá-los. Quinze pares foram apresentados para cada um dos níveis de força associativa. Na segunda etapa, 15 sentenças eram apresentadas como pista, durante 5 segundos cada uma. As pistas tinham a seguinte forma "Um exemplo de mobília é _____. A tarefa dos participantes era produzir o primeiro exemplo da categoria que ela poderia lembrar da lista estudada na primeira etapa do experimento.

A tarefa de julgamentos de tipicidade foi realizada após a produção lingüística. O tempo para realizar a tarefa era livre. As categorias e os exemplos foram apresentados impressos em papel, tendo sido elaboradas 5 ordens randômicas. Cada versão do instrumento incluía o nome da categoria e os 3 exemplos, um de cada nível de força associativa, em blocos, no seguinte formato:

\section{Mobília: mesa ( ) cama ( ) cômoda ( )}

As instruções da tarefa solicitavam que os participantes avaliassem o grau de qualidade de cada item como um representante da categoria, em uma escala que variava de 1 a 7. Nesta escala, números menores indicavam que o membro era um exemplo melhor do que números maiores. Para realizar os julgamentos, os participantes deveriam escrever, entre parênteses, o número que melhor correspondesse à sua percepção da representatividade de cada membro da respectiva categoria.

\section{Resultados}

Uma análise variância para medidas repetidas indicou que a força associativa teve um efeito significativo na produção lingüística, adotando-se um nível de significância de $0,05[\mathrm{~F}(2,60)=5,23, \mathrm{MSe}=5,73]$. Os resultados mostraram que membros mais fortemente associados às suas categorias foram produzidos com maior probabilidade do que membros fracamente associados. As médias verificadas foram 5,39, 4,97 e 3,52 para os níveis, forte, médio e fraco, respectivamente. Teste subseqüente para avaliar as diferenças entre as médias (Fisher LSD $=1,26$, bicaudal) indicou que, excetuando a diferença entre as médias forte $\mathrm{e}$ médio, as demais foram significativas.

A força associativa também influenciou significativamente os julgamentos de tipicidade. Os resultados indicaram que membros mais fortemente associados às suas categorias foram julgados como melhores exemplos do que membros fracamente associados, $\mathrm{F}(2,30)=22,24, \mathrm{MSe}=$ 0,46. As médias das tipicidades foram 1,56, 2,10 e 3,13 para os membros associados forte, média e fracamente às suas categorias. Teste subseqüente evidenciou (Fisher LSD $=0,53$, bicaudal) que todas as diferenças entre as médias foram significativas.

\section{Discussão}

Este estudo pretendeu apresentar um procedimento para avaliar a participação dos atributos nos julgamentos de tipicidade, minimizando a influência da memória para esta informação, e estender a hipótese da acessibilidade conceitual (Janczura \& Nelson, 1999) para a tarefa de produção lingüística envolvendo categorias e seus membros.

Demonstrou-se que a força associativa influencia significativamente a produção lingüística e os julgamentos de tipicidade. Níveis diferentes de acessibilidade conceitual puderem prever a probabilidade de um membro ser gerado como exemplo de uma categoria no contex to de sentenças. Membros de categorias mais acessíveis na memória estão associados com melhores níveis de desempenho do que exemplos menos acessíveis.

Além disto, um procedimento foi proposto para controlar a quantidade de informação que o indivíduo precisa recuperar 
da memória de longo-prazo quando é solicitado a estimar a tipicidade de membros de categorias. Neste procedimento, a informação é disponibilizada ao indivíduo ao invés deste ter que evocá-la para diferentes categorias. Os resultados indicaram que os graus de tipicidade foram previstos pela força da associação entre categorias e seus membros.

Nestas condições, as variações na acessibilidade dos conceitos foram capazes de explicar as variações nos julgamentos de tipicidade. Ou seja, exemplos mais fortemente associados às suas categorias foram julgados como melhores exemplos do que membros fracamente associados às mesmas. Este padrão de resultados tem sido observado em diferentes estudos (Janczura, 1994; Janczura \& Nelson, 1999). Argumenta-se que julgamentos de tipicidade deveriam ser re-interpretados como indicadores da acessibilidade conceitual similarmente a outras medidas diretas de memória como o reconhecimento e a evocação, e que estas medidas revelam a aprendizagem anterior e a experiência episódica com categorias e seus membros.

Entretanto, esta re-interpretação se aplica somente para categorias conhecidas, isto é, categorias com as quais os indivíduos têm alguma experiência. A hipótese da acessibilidade conceitual aplicada aos julgamentos de tipicidade não se sustenta para categorias novas ou artificiais porque, neste caso, não houve a oportunidade para a acessibilidade se desenvolver.

A literatura tem extensivamente demonstrado que a percepção da representatividade é baseada na estrutura do estímulo quando um item é novo ou foi produzido artificialmente no laboratório. É provável que a informação sobre a estrutura da categoria desempenhe um papel mais central, dirigindo a percepção da tipicidade, quando o exemplo a ser julgado é novo ou é membro de uma categoria pobremente definida. Entretanto, é importante destacar que ainda não foi resolvida a questão sobre quais são os atributos ou características das categorias naturais que os indivíduos consideram quando engajados em tarefas de tipicidade. Além disto, alguns estudos têm questionado a importância da tipicidade baseada na noção de similaridade em tarefas que envolvem a categorização e o raciocínio (Bailenson, Shum, Atran, Medin \& Coley, 2002).

A relação teórica entre a experiência e a acessibilidade conceitual, e entre a acessibilidade e medidas de força associativa, tipicidade, probabilidade de evocação e reconhecimento e ordem da evocação é direta: a acessibilidade dos conceitos na memória está determinada tanto pela experiência prévia quanto aprendizagem episódica. Este arcabouço sugere que estes testes representam diferentes maneiras de avaliar a acessibilidade da informação conceitual na memória.

É mais provável que os indivíduos tenham experienciado, durante o seu desenvolvimento, o par fru$\mathrm{ta} /$ maçã mais frequentemente do que fruta/tomate. Diferentes níveis de repetição de pares categoria/exemplo tornam os membros diferentemente associados às suas categorias. A hipótese da acessibilidade conceitual sustenta que exemplos experimentados mais frequentemente desenvol- vem associações mais fortes com suas categorias do que exemplos experimentados menos frequentemente. A força associativa de um membro em relação a sua categoria é revelada, descritivamente, pela probabilidade daquele ser produzido em normas de associação livre. Os membros que são mais fortemente associados a esta representação mental são mais prováveis de serem produzidos como itens de uma categoria, lembrados com maior probabilidade, e percebidos como melhores exemplos do que membros fracamente associados às categorias.

\section{Referências}

Ashcraft, M. H. (1978). Property norms for typical and atypical items from 17 categories. Memory E Cognition, 6, 227-232.

Bailenson, J. N., Shum, M. S., Atran, S., Medin, D. L., \& Coley, J. D. (2002). A bird's eye view: Biological categorization and reasoning within and across cultures. Cognition, 84, 1-53.

Barsalou, L.W. (1985). Ideals, central tendency, and frequency of instantiation as determinants of typicality effects. Journal of Experimental Psychology: Learning, Memory, and Cognition, 11, 629-649.

Barsalou, L.W. (1987). The instability of graded structure: Implications for the nature of concepts. In U. Neisser (Ed.), Concepts and conceptual structure - Ecological and intellectual factors in categorization (pp. 101-140). Cambridge, UK: Cambridge University Press.

Barsalou, L.W., \& Sewell, D. R. (1984). Constructing representations of categories from different points of view (Tech. Rep. No. 2). In U. Neisser (Ed.), Concepts and conceptual structure - Ecological and intellectual factors in categorization (pp. 101-140). Cambridge, UK: Cambridge University Press.

Battig, W. F., \& Montague, W. E. (1969). Category norms for verbal items in 56 categories: A replication and extension of the Connecticut category norms. Journal of Experimental Psychology Monographs, 80, 1-46.

Begg, I., \& Armour, V. (1991). Repetition and the ring of truth: Biasing comments. Canadian Journal of Behavior Science, 23, 195-2 13 .

Estes, Z. (2003). Domain differences in the structure of artifactual and natural categories. Memory and Cognition, 31, 199-2 14.

Evans, D., Herbert, J., Nelson, G., Rosemary, O., \& Gaudiano, B. A. (2002). Determinants of diagnostic prototypicality judgments of the personality disorders. Journal of Personality Disorders, 16, 95-106.

Hampton, J. A. (1979). Polymorphous concepts in semantic memory. Journal of Verbal Learning and Verbal Behavior, 18, 441-461.

Hekkert, P., Snelders, D., \& van-Wieringen, P. C. W. (2003). 'Most advanced, yet acceptable': Typicality and novelty as joint predictors of aesthetic preference in industrial design. British Journal of Psychology, 94, 111-124.

Janczura, G. A. (1994). Associative strength and frequency of instantiation as determinants of typicality effects. Unpublished doctoral dissertation, University of South Florida, St. Petersburg, United States.

Janczura, G. A. (1996). Normas associativas para 69 categorias semânticas. Psicologia: Teoria e Pesquisa, 12, 237-244.

Janczura, G. A., \& Nelson, D. L. (1999). Concept accessibility as the determinant of typicality judgments. American Journal of Psychology, 112, 1-19. 
Kahneman, D., \& Tversky, A. (1973). On the psychology of prediction. Psychological Review, 80, 237-251.

Kiran, S., \& Thompson, C. K. (2003). Effect of typicality on online category verification of animate category exemplars in aphasia. Brain and Language, 85, 441-450.

McCloskey, M., \& Glucksberg, S. (1978). Natural categories: Well-defined or fuzzy sets? Memory \& Cognition, 6, 462-472.

McEvoy, C. L., \& Nelson, D. L. (1982). Category name and instance norms for 106 categories of various sizes. American Journal of Psychology, 95, 581-634.

Medin, D. L. (1989). Concepts and conceptual structure. American Psychologist, 44, 1469-1481.

Medin, D. L., \& Schaffer, M. M. (1978). Context theory of classification learning. Psychological Review, 85, 207-238.

Mervis, C. B., Catlin, J., \& Rosch, E. (1976). Relationship among goodness-of-example, category norms, and word frequency. Bulletin of the Psychonomic Society, 7, 283-284.

Murphy, G. L., \& Medin, D. L. (1985). The role of theories in conceptual coherence. Psychological Review, 92, 289-316.

Nelson, D. L., \& McEvoy, C.L. (1976). Rhyme category norms. Unpublished manuscript, University of South Florida, St. Petersburg.

Nelson, D. L., McEvoy, C. L., \& Dennis, S. (2000). What is free association and what does it measure. Memory and Cognition, 28, 887-899.

Op-de-Beeck, H., \& Wagemans, J. (2001). Visual object categorisation at distinct levels of abstraction: A new stimulus set. Perception, 30, 1337-1361.

Rips, L. J., Shoben, E. J., \& Smith, E. E. (1973). Semantic distance and the verification of semantic relations. Journal of Verbal Learning and Verbal Behavior, 12, 1-20.

Rosch, E. (1975). Cognitive representations of semantic categories. Journal of Experimental Psychology: General, 104, 192-233.

Rosch, E., \& Mervis, C. B. (1975). Family resemblance: Studies in the internal structure of categories. Cognitive Psychology, 7, 573-605.

Roth, E. M., \& Shoben, E. J. (1983). The effect of context on the structure of categories. Cognitive Psychology, 15, 346-378.

Shortess, G. K., Clarke, J. C., Richter, M. L., \& Seay, M. (2000). Abstract or realistic? Prototypicality of paintings.Visual Arts Research, 26, 70-79.

Smith, E. E., Shoben, E. J., \& Rips, L. J. (1974). Structure and processes in semantic memory: A featural model for semantic decisions. Psychological Review, 81, 2 14-241.

Verbeemen, T., Storms, G., \& Verguts, T. (2003). Determinants of speeded categorization in natural concepts. Psychologica Belgica, 43, 139-151.
Veryzer, R. W., \& Hutchinson, J. W. (1998). The influence of unity and prototypicality on aesthetic responses to new product designs. Journal of Consumer Research, 24, 374-394.

Whitfield, T. W. A., \& Slatter, P. E. (1979). The effects of categorization and prototypicality on aesthetic choice in a furniture selection task. British Journal of Psychology, 70, 65-75.

Whittlesea, B.W.A. (1993). Illusions of familiarity. Journal of Experimental Psychology: Learning, Memory, and Cognition, 19, 1235-1253.

Wood, C. (1971). Wood's unabridged rhyming dictionary. New York: World.

Zhang, J., \& Chen, J. (2002). The effects of ways of linguistic expression on the semantic recovery time of natural concepts. Psychological Science China, 25, 40-42, 36.

Recebido: 17/01/2005

$1^{a}$ revisão: $04 / 08 / 2005$

$2^{a}$ revisão: $15 / 03 / 2006$

Aceite final: 23/03/2006

\section{Anexo A}

Materiais utilizados no Experimento 1:

Categorias, Membros, Atributos

Flor: Tulipa, Orquídea, Margarida (terra, talo, folhas, colorida, caras, delicada).

Cobra Venenosa: Coral, Quatiara, Jararaca (troca de pele, nojenta, assustadora, colorida, fina, rasteira).

Calçado: Sapato, Sandália, Botina (confortável, aquece os pés, pode ser de tecido, caro(a), palmilha, couro).

Ser Místico: Duende, Guru, Deus (espiritualidade, inteligente, mito, poder, religião, onipotente).

Bebida Alcoólica: Licor, Vinho, Vodka (destilado(a), transparente, forte, embriaga, álcool, cheiro agradável).

Ferramenta de Carpinteiro: Pá, Serrote, Esmeril (para construção, ferro, grande, perigoso(a), pequeno(a), barulho).

Mobília: Cama, Cômoda, Mesa (redonda, confortável, madeira, ferro, dura, quadrado(a).

Instrumento de Percussão: Cuíca, Atabaque, Tambor (barulho, som diferente, tocado(a) com as mãos, arredondado(a), ritmo, vários tamanhos).

Raça de Cachorro: Pastor Alemão, Poodle, Collie (boca, brincalhão, forte, dócil, fiel, coleira). 